\title{
The periods of the $\beta$ Cephei star $\kappa$ Scorpii as observed by WIRE
}

\author{
J. Cuypers ${ }^{1}$, D. Buzasi ${ }^{2}$, K. Uytterhoeven ${ }^{3}$ \\ ${ }^{1}$ Koninklijke Sterrenwacht van België, Ringlaan 3, B-1180, Brussels, \\ Belgium \\ ${ }^{2}$ Department of Physics, US Air Force Academy, Colorado Springs, CO \\ 80840 , USA \\ ${ }^{3}$ Instituut voor Sterrenkunde, Katholieke Universiteit Leuven, \\ Celestijnenlaan 200 B, B-3001 Leuven, Belgium
}

\begin{abstract}
The $\beta$ Cephei star $\kappa$ Scorpii is known to be multiperiodic from old photometric observations. A main period of $4.8 \mathrm{hr}$ was detected, and from a beat period of about $7 \mathrm{~d}$ a secondary period of $4.9 \mathrm{hr}$ was calculated. These periods were also found in recently analyzed line profile data. In two short data sets (separated by $152 \mathrm{~d}$ ) obtained by the star camera of the satellite WIRE (Wide-field Infrared Explorer) the known main period of $4.8 \mathrm{hr}$ is clearly present. The secondary period could also be confirmed, although some other periodicities are present as well. While the amplitude of the main variation is about $0.0055 \mathrm{mag}$, the amplitude of the secondary is only 0.0014 mag. Two longer periods ( 3.6 and $0.45 \mathrm{~d}$ ) and a period of $4.2 \mathrm{hr}$ were also found significant in the WIRE data.
\end{abstract}

\section{Introduction}

$\kappa$ Scorpii (HR 6580, HD 160578, HIP 86670, $m_{V}=2.41$, B1.5III) is known to be a $\beta$ Cephei star from photometric observations. The star is also a spectroscopic binary and the orbital parameters were determined by De Mey (1997) and refined by Uytterhoeven et al. (2001). The orbital period is $195.65 \mathrm{~d}$, the eccentricity 0.49 and the mass function is $1.49 \mathrm{M}_{\odot}$.

Multiperiodicity in the light variations of $\kappa$ Scorpii was detected by Shobbrook \& Lomb (1972) and confirmed by Lomb \& Shobbrook (1975). A beat period of $7 \mathrm{~d}$ was noticed and this led to at least one other frequency $f_{2}=$ $4.8678381 \mathrm{~d}^{-1}$ besides the main frequency: $f_{1}=5.0042461 \mathrm{~d}^{-1}$. Since unrealistic errors were claimed for these frequencies, amplitude and frequency variations were thought to be present, and the general frequency structure was unclear, we intend to re-analyze these data soon.

Aerts et al. (1989) noticed line profile variability for this star. Uytterhoeven et al. (2001) confirmed the frequency $f_{1}$ and found some indications of $f_{2}$ in the line profile variations, but not all variability could be satisfactorily described. A tentative mode identification gave for $f_{1}(\ell=1, m=-1)$ or $(\ell=2, m=-2)$ while $f_{2}$ seemed to be a higher order $(\ell \geq 4)$ tesseral mode. 


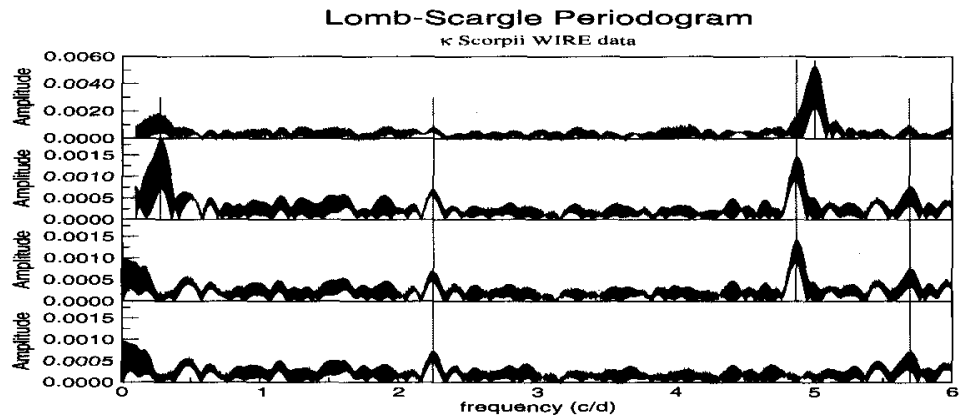

Figure 1. Periodogram of the WIRE data with consecutive prewhitening. The positions of the 5 frequencies are indicated.

\section{The satellite WIRE}

The Wide-Field Infrared Explorer (WIRE) mission was launched by NASA on 1999 March 4. The primary mission failed several days after launch, but conversion of the spacecraft to an asteroseismology platform, using the onboard star camera as detector, was very successful. WIRE began to operate in this mode on 1999 April 30 and continued until 2000 September 30. A new run of observations started in 2003.

Buzasi (2002) and others reported on the asteroseismological results of WIRE in general. Results on individual bright variable stars are already published: e.g. on the $\delta$ Sct $\operatorname{star} \theta^{2}$ Tau by Poretti et al. (2001), on the $\beta$ Cep star $\beta$ Cru by Cuypers et al. (2002), on the red giant Arcturus (Retter et al. 2003). In Buzasi (2003) the spacecraft pointing precision and some of the instrumental effects, such as scattered light and analogue-to-digital converter errors, are discussed.

\section{WIRE observations of $\kappa$ Sco}

In total, 911978 observations of $\kappa$ Sco were available from WIRE. These observations came in two blocks, one of $10 \mathrm{~d}$ and another of $5 \mathrm{~d}$, separated by a gap of $152 \mathrm{~d}$. This long interruption causes a typical pattern of peaks in periodograms, all separated by the corresponding $\Delta f=0.0066 \mathrm{~d}^{-1}$. As a consequence all frequencies have several equally significant aliases at this distance in frequency. We carried out a frequency analysis with such classical methods as Lomb-Scargle periodograms (Fig. 1) and least squares harmonic fitting applied to the unbinned data, to the data binned per 50 s (i.e., approximately per 100 observations, resulting in 9289 observations), and to data with only 2 binned observations per orbit (351 observations). We also used a program for direct search of multiple frequencies without prewhitening or initial values as discussed by Schoenaers \& 

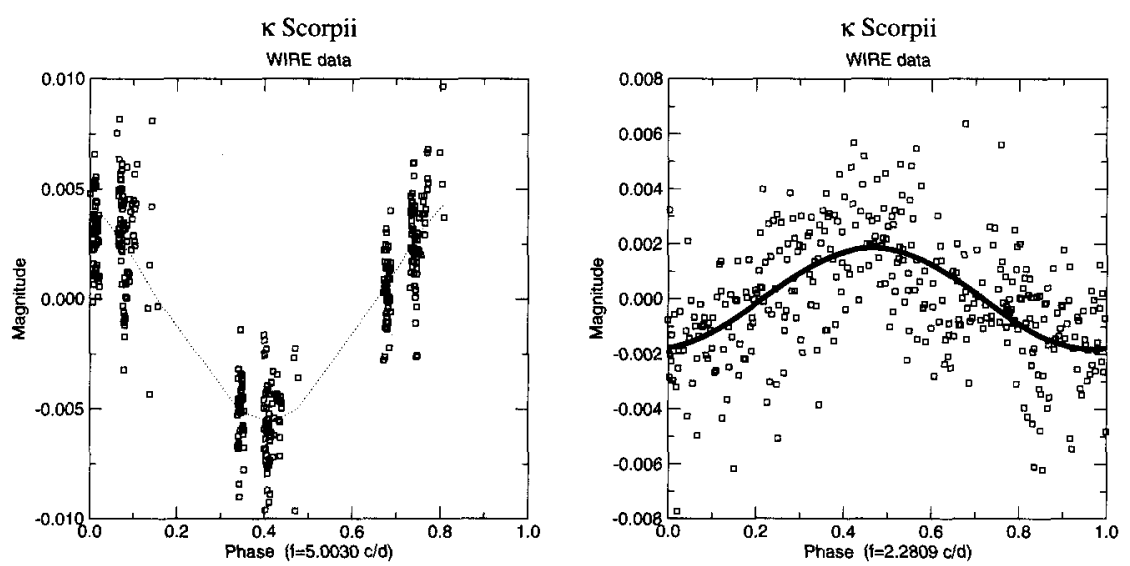

Figure 2. Phase diagrams for the frequencies $f_{1}$ (left) and $f_{3}$ (right).

Cuypers (2004, these proceedings). All methods gave the same results on the WIRE data. The primary frequency is clearly present: $f_{1}=5.0030 \mathrm{~d}^{-1}$ (amplitude $0.0055 \mathrm{mag}$ ). The theoretical error on this frequency is $0.0006 \mathrm{~d}^{-1}$, but the period corresponding to $f_{1}$ is unfortunately close to three times the orbital period of the spacecraft. The odd distribution in the phase diagram (Fig. 2a) will surely introduce extra error into the frequency determination. The next frequency is not found at the photometric frequency $f_{2}$, but at $f_{3}=0.2809 \mathrm{~d}^{-1}$ (Fig. 2b) with an amplitude of $0.0018 \mathrm{mag}$. The photometric frequency $f_{2}$ shows up at $4.8710 \mathrm{~d}^{-1}$ (Fig. 3a), but with an amplitude of only 0.0014 mag. Every frequency has also its alias frequencies caused by the spacecraft's orbital period of $5750.4 \mathrm{~s}$ (or $15.025 \mathrm{~d}^{-1}$ ). Two other frequencies appear at the limit of detection: $f_{4}=2.2498 \mathrm{~d}^{-1}$ (amplitude $0.0008 \mathrm{mag}$ ) and $f_{5}=5.6950 \mathrm{~d}^{-1}$ (Fig. 3b) with an amplitude of 0.0007 mag.

\section{Discussion and conclusions}

At least three frequencies are detected in the WIRE data, two more are significant as well. At the moment we have no explanation for frequencies $f_{3}$ and $f_{4}$. Their corresponding periods are too large (or their aliases too small) to be typical $\beta$ Cephei frequencies. A rotation period is not very likely. One could speculate that the periods come from SPB-like variations in the secondary component, but with a mass estimate of $12 \mathrm{M}_{\odot}$ (Uytterhoeven et al. 2001) this is not very likely either.

The amplitudes of $f_{1}$ and $f_{2}$ are not in contradiction with the mode identifications of the line profiles. An $\ell=1$ mode was associated with $f_{1}$, so a higher amplitude in the light variations was expected and is observed. For other frequencies such as $f_{2}$, higher $\ell$ values $(\ell \geq 4)$ were identified but, as a consequence, smaller amplitudes in the light variations are not surprising. But to make the mode identification more reliable, a more detailed comparison with the line profile data and the old photometric data is necessary. 

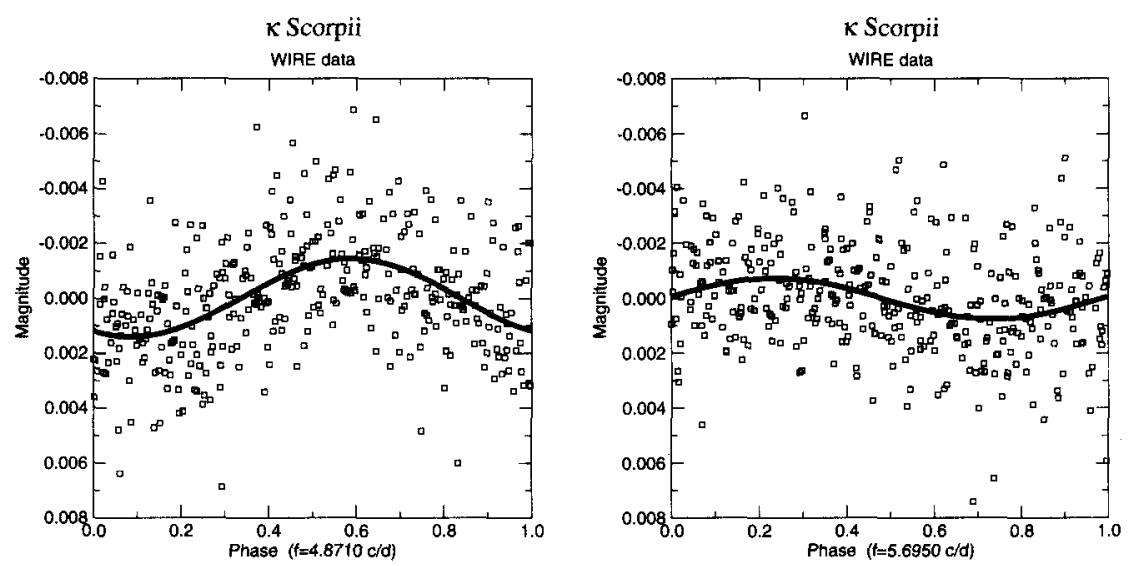

Figure 3. Phase diagrams for the frequencies $f_{2}$ (left) and $f_{5}$ (right).

These results once more illustrate how high-precision photometry can be successfully accomplished with a completely uncalibrated instrument originally designed for a totally different purpose.

Acknowledgments. JC and KU acknowledge support from Research Programme G.0178.02 of the Fund for Scientific Research - Flanders (Belgium).

\section{References}

Aerts, C., Balona, L.A., Waelkens, C. 1990, in ASP Conf. Ser., Vol. 11, Confrontation Between Stellar Pulsation and Evolution, eds C. Cacciari \& G. Clementini (San Francisco: ASP), 290

Buzasi, D. 2002, in ASP Conf. Ser., Vol. 259, Radial and Nonradial Pulsations as Probes of Stellar Physics, eds C. Aerts, T. Bedding, \& J. ChristensenDalsgaard (San Francisco: ASP), 616

Buzasi, D. 2003, in 2nd Eddington Workshop, Stellar structure and habitable planet finding, ESA, in press

Cuypers, J., Aerts, C., Buzasi, D., Catanzarite, J., Conrow, T., Laher, R. 2002, A\&A, 392, 599

De Mey, K. 1997, Ph. Thesis, Katholieke Universiteit Leuven

Lomb, N.R. Shobbrook, R. 1975, MNRAS, 173, 709

Poretti, E., Buzasi, D., Laher, R., Catanzarite, J., Conrow, T. 2001, A\&A, 382, 157

Retter, A., Bedding, T., Buzasi, D., Kjeldsen, H., Kiss, L. 2003, ApJ, 591, L151 Shobbrook, R., Lomb, N.R. 1972, MNRAS, 156, 181

Uytterhoeven, K., Aerts, C., De Cat, P., De Mey, K., Telting, J. H., Schrijvers, C., De Ridder, J., Daems, K., Meeus, G., Waelkens, C. 2001, A\&A, 371, 1035 